\title{
ANÁLISE DA DINÂMICA DO SISTEMA DE PRODUÇÃO VOCAL NA PRESENÇA DE PATOLOGIAS DA LARINGE
}

\author{
Washington C. De A. Costa*, Silvana C. Costa*, Vinícius J. D. Vieira*, Clara F. Dourado*, \\ Tiago B. P. ARAújo* \\ *Instituto Federal de Educação, Ciência e Tecnologia da Paraíba, João Pessoa, Paraíba, Brasil
}

Emails: washington@ifpb.edu.br, silvana@ifpb.edu.br, viniciusjdv@gmail.com, clarafigueiredo.ee@gmail.com, tiagobparaujo@gmail.com

\begin{abstract}
This work evaluates the use of measures obtained from nonlinear dynamic analysis in discrimination between healthy voices and voices affected by laryngeal pathologies (Reinke's edema, nodules and vocal fold paralysis). The employed measures, obtained by recurrence quantification analysis, are: transitivity, mean diagonal line length and recurrence time of types 1 and 2 . The results of the classification process suggest that the evaluated measures have discriminative power between the two considered groups, and can be used in diagnosis auxiliary systems of laryngeal pathologies.
\end{abstract}

Keywords - Dynamical systems, digital processing of speech signal, laryngeal pathologies, recurrence quantification analyisis.

\begin{abstract}
Resumo - Este trabalho trata da avaliação do uso de medidas obtidas a partir da análise dinâmica não linear na discriminação entre vozes saudáveis e vozes afetadas por patologias laríngeas (edemas de Reinke, nódulos e paralisia nas pregas vocais). As medidas empregadas, obtidas por meio da análise de quantificação de recorrência, são: transitividade, comprimento médio das linhas diagonais e o tempo de recorrência dos tipos 1 e 2 . Os resultados obtidos no processo de classificação indicam que as medidas avaliadas apresentam potencial discriminativo entre os dois grupos de sinais de voz considerados, podendo ser empregadas em sistemas de auxílio ao diagnóstico de patologias na laringe.
\end{abstract}

Palavras-chave - Sistemas dinâmicos, processamento digital de sinais de voz, patologias da laringe, análise de quantificação de recorrência.

\section{Introdução}

A voz é uma emissão acústica voluntária, considerada principal ferramenta de comunicação humana (Behlau, 2001). Para uma boa produção vocal é necessária uma voz saudável. A qualidade vocal relaciona-se com a frequência, a amplitude e a composição dos harmônicos da onda sonora produzida, bem como ao modo de vibração das pregas vogais, o tamanho e o formato do trato vocal, a tensão e o tônus das paredes faríngeas, podendo ser modificada por fatores anatômicos ou patológicos (Fant, 2004).

Há uma grande variedade de doenças relacionadas à laringe que afetam a qualidade vocal, prejudicando a comunicação humana. Essas patologias podem ser tanto de origem orgânica como nódulos, cistos ou edemas, quanto de origem neurológica, tal como paralisia nas pregas vocais. Algumas dessas patologias como pólipos, nódulos e edemas de Reinke, por exemplo, ocorrem, principalmente, devido a hábitos sociais não-saudáveis - como tabagismo e alcoolismo - e ao abuso vocal. No caso de paralisia, as principais causas incluem traumas cirúrgicos ou acidentais, doença cardiovascular e doenças neurológicas (Stemple et al., 2000).

Métodos tradicionais de avaliação e diagnóstico de doenças da laringe são realizados, em geral, por meio da escuta da voz do paciente e da inspeção visual das pregas vocais por exames de videolaringoscopia, com ótica telescópica ou fibra ótica acoplada a um sistema de vídeo. Tais métodos, no entanto, requerem um profissional experiente e, em alguns casos, são considerados invasivos, causando certo desconforto ao paciente.

A análise acústica, utilizando técnicas de processamento digital de sinais, pode ser usada como ferramenta não invasiva de apoio ao diagnóstico médico e acompanhamento de tratamentos e terapias vocais, de forma objetiva. Essa ferramenta não substitui os métodos tradicionais, mas pode auxiliar no diagnóstico, reduzindo a quantidade de exames videolaringoscópicos, podendo, ainda, ser efetuado à distância. A precisão do diagnóstico, contudo, depende da escolha das características e parâmetros da fala que melhor representem a desordem vocal provocada por uma determinada patologia.

\section{Propósito}

Do ponto de vista dos sistemas dinâmicos, um sinal de voz pode ser considerado como uma observação do sistema de produção vocal, que pode ser usada para revelar e modelar sua dinâmica usando técnicas lineares ou não lineares. No primeiro caso, considerando um modelo linear de produção de fala, é possível representar, de forma geral, o sistema de produção de voz como um sistema fonte-filtro, no qual as pregas vocais são consideradas a fonte sonora e o trato vocal, o filtro. O sinal resultante é o sinal acústico da voz. 
Para muitas aplicações do processamento digital de fala, como a codificação, síntese, e reconhecimento, um bom desempenho pode ser conseguido com o modelo fonte-filtro, o qual reflete as características gerais de padrões de tempo e articulatórios, bem como as propriedades de variação da frequência. Por outro lado, métodos mais detalhados de modelagem de produção de fala, com base em características físicas reais, são necessários para áreas como análise do movimento das pregas vocais, efeitos de uma patologia sobre a produção da fala, e outras áreas de aplicação (Deller et al., 2000).

Existem várias não linearidades envolvidas na vibração das pregas vocais e na geração da onda glotal (de Oliveira Rosa, 2002). Estas incluem efeitos devidos às fortes forças de restauração na colisão das pregas vocais, turbulência no fluxo de ar devido a uma constrição do trato vocal durante a produção de sons surdos e ao acoplamento não linear entre a fonte e o trato vocal durante a pronúncia de alguns sons sonoros (Kumar and Mullick, 1996). Devido a tais fatores, os métodos clássicos de análise de dados baseados em um modelo linear têm sido enriquecidos com novos métodos que são derivados da teoria dos sistemas dinâmicos não lineares (Jiang et al., 2006).

Os gráficos de recorrência (Eckmann et al., 1987) têm surgido, nas duas últimas décadas, como uma técnica de análise não linear para o estudo da dinâmica de sistemas dissipativos. Embora pequenas perturbações em tais sistemas causem divergência exponencialmente crescente entre os estados, após algum tempo o sistema retorna a um estado que é arbitrariamente próximo a um estado precedente e passa por uma evolução similar. Os gráficos de recorrência permitem visualizar o comportamento recorrente dos sistemas dinâmicos de baixa ou de alta dimensionalidade.

Este trabalho é realizado tendo como principal objetivo a identificação de um conjunto de características, obtidas a partir de medidas relacionadas com a análise dinâmica não linear, que seja capaz de caracterizar e discriminar, de forma eficiente, sinais de vozes saudáveis e vozes afetadas por diferentes tipos de patologias nas pregas vocais (edema de Reinke, nódulos ou paralisia). Para tanto, é analisado o comportamento de sinais de voz por meio de medidas quantitativas, as chamadas medidas de quantificação de recorrência (MQR), extraídas dos gráficos de recorrência.

\section{Métodos}

A análise dinâmica de sinais pode ser feita por meio de uma modelagem matemática ou por meio da análise de séries temporais. A primeira técnica implica na tentativa de se construir um modelo que possa ser usado para se obter informações úteis associadas à produção do fenômeno em es- tudo. Por outro lado, a análise de séries temporais considera simplesmente uma série temporal escalar, em geral, associada com uma aquisição experimental para entender o comportamento dinâmico do sistema. O ponto essencial dessa análise é que uma série temporal contém informações sobre variáveis não observáveis do sistema, o que permite a reconstrução do espaço de estados (Savi, 2006).

\subsection{Reconstrução do espaço de estados}

A técnica de reconstrução do espaço de estados é baseada no teorema da imersão de Takens (Takens, 1981). Este teorema permite reconstruir um espaço de estado $m$-dimensional similar ao espaço de estado original, $d$-dimensional, a partir de uma única variável de estado, no caso, o próprio sinal de voz. Assim, vetores $\vec{\xi}_{i} m$-dimensionais são reconstruídos a partir da série temporal (sinal de voz) $\left\{x_{i}\right\}$, em que $x_{i}=x\left(t_{i}\right), i=1, \ldots, T$, e de suas $m-1$ versões defasadas no tempo,

$$
\vec{\xi}_{i}=\left\{x\left(t_{i}\right), x\left(t_{i}+\tau\right), \ldots, x\left(t_{i}+(m-1) \tau\right)\right\}
$$

em que $m$ é a chamada dimensão de imersão e $\tau$ é o passo de reconstrução ou tempo de defasagem.

O método de Takens é também chamado método dos atrasos temporais e permite a reconstrução do atrator. Embora o atrator reconstruído não seja idêntico ao original, pode-se demonstrar que as propriedades topológicas são preservadas (difeomorfismo). Além disso, a série temporal de uma única variável é suficiente para a reconstrução desde que a dimensão de imersão seja suficientemente grande (Takens, 1981).

Para um número infinito de pontos e na ausência de ruído, a escolha do passo de reconstrução $\tau$ é, na grande maioria dos casos, arbitrária (Takens, 1981). Entretanto, as séries temporais experimentais são finitas, usualmente contaminadas com ruído externo e obtidas com o uso de filtros. Nessa situação a reconstrução depende, e muito, da escolha correta do passo (FiedlerFerrara and do Prado, 1994). O método baseado na função de informação mútua (Fraser and Swinney, 1986) foi o método utilizado neste trabalho para a determinação do tempo de defasagem ou passo de reconstrução.

A dimensão $m$ do espaço de fases reconstruído não é necessariamente idêntica à dimensão $d$ do espaço de fases real dos vetores que representam a dinâmica do sistema físico. Em geral é necessário reconstruir-se o atrator em espaços de fases com dimensão suficientemente elevada ( $m \geq 2 D_{0}+1$, onde $D_{0}$ é a dimensão de Hausdorff (Hausdorff, 1919) do atrator) para que se tenha segurança com relação aos resultados; caso contrário, o atrator aparecerá dobrado sobre si mesmo como numa projeção. O método das Falsas 
Vizinhanças ou Método dos Falsos Vizinhos Próximos (Kennel et al., 1992) foi o método utilizado neste trabalho para a determinação da dimensão mínima de imersão.

\subsection{Gráficos de Recorrência e suas Medidas de Quantificação}

Vários métodos de análise têm tentado estimar medidas e propriedades não lineares de processos naturais. Entretanto, a maioria dos métodos da análise não linear exigem séries um tanto longas ou estacionaridade (Marwan, 2003).

Recentemente, outro método baseado na análise não linear de dados tem sido usado: os Gráficos de Recorrência (ou Recurrence Plots - RP) criados como método de análise de sistemas dinâmicos por Eckmann et al. (Eckmann et al., 1987). A ideia de recorrência surgiu no século XIX, a partir do Teorema da Recorrência, do físico e filósofo francês J. H. Poincaré (1890).

Recorrência é uma propriedade fundamental dos sistemas dinâmicos dissipativos e a principal vantagem dos gráficos de recorrência é a possibilidade do uso dessa técnica para análise de séries curtas e não-estacionárias (Marwan, 2003; Marwan and Kurths, 2004).

O primeiro passo para a construção do gráfico de recorrência de uma série temporal de comprimento $T$ é a imersão dessa série em um espaço $m$-dimensional, em geral, por meio do método dos atrasos temporais (Takens, 1981). Daí são construídos $N$ vetores (Eq. 1), de dimensão $m$, representativos da dinâmica do sistema em análise no espaço de fase.

De acordo com a definição originalmente dada em (Eckmann et al., 1987), o gráfico de recorrência é uma matriz $N \times N$ (matriz gráfica), em que $N$ é o número de vetores (estados do sistema) de dimensão $m$, preenchida por pontos brancos e pretos. O ponto preto, chamado de ponto recorrente, é colocado na matriz de recorrência nas coordenadas $(i, j)$ somente se a distância $d(i, j)$ nos instantes $n=i$ e $n=j$ (entre o estado corrente do sistema e o estado a ser comparado) for menor que certa distância (raio) $\varepsilon$, fixada no centro do estado corrente.

Uma definição alternativa comumente utilizada para o Gráfico de Recorrência é dada por (Marwan, 2003):

$\mathcal{R}_{i, j}^{m, \varepsilon}=\Theta\left(\varepsilon-\left\|\vec{\xi}_{i}-\vec{\xi}_{j}\right\|\right), \quad \vec{\xi}_{i} \in R^{m}, \quad i, j=1 \ldots N$,

em que:

- $N$ é o número de estados $\vec{\xi}_{i}$ considerados;

- $\varepsilon$ é o raio da vizinhança (threshold) no ponto $\vec{\xi}_{i}$

- \| - || é a norma da vizinhança, comumente a norma euclidiana;
- $\Theta(\cdot)$ é a função de Heaviside;

- $m$ é a dimensão de imersão (graus de liberdade).

Pontos isolados, linhas diagonais, como também linhas verticais e horizontais (e a combinação destas para formar estruturas variadas) são estruturas típicas presentes nos gráficos de recorrência (Marwan and Kurths, 2004).

A análise visual dos gráficos de recorrência fornece resultados de forma qualitativa. Por exemplo, processos com comportamento estocástico tendem a não apresentar estruturas diagonais (Figura 1). Por outro lado, processos determinísticos causam diagonais mais longas e menos pontos de recorrência isolados (Figura 2). Nas Figuras 3 e 4 pode ser observada a dinâmica de um sinal de voz saudável e de um sinal de voz patológica (edema), respectivamente.

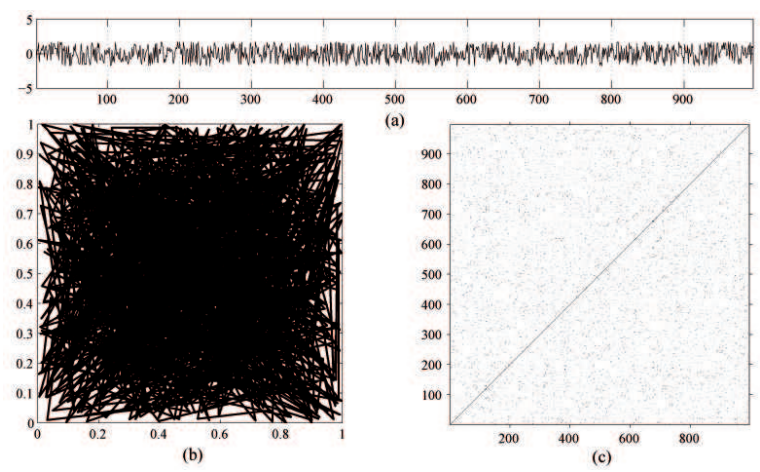

Figura 1: Ruído branco (a) Série temporal, (b) Retrato de fase e (c) Gráfico de recorrência; $T=$ 1.000, $m=3, \tau=1$ e $\varepsilon=0,1$ (norma euclidiana).

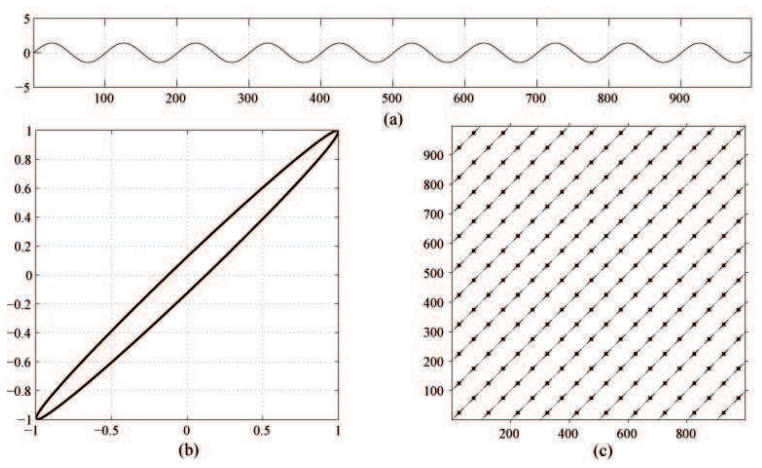

Figura 2: Sinal senoidal (a) Série temporal, (b) Retrato de fase e (c) Gráfico de recorrência; $T=$ 1.000, $m=2, \tau=4$ e $\varepsilon=0,1$ (norma euclidiana).

A avaliação por meio dos gráficos de recorrência é subjetiva, e pode levar a mais de um diagnóstico, dependendo do profissional que analisa a voz do paciente.

Como forma de quantificar o comportamento do sistema, facilitando a análise dos da- 

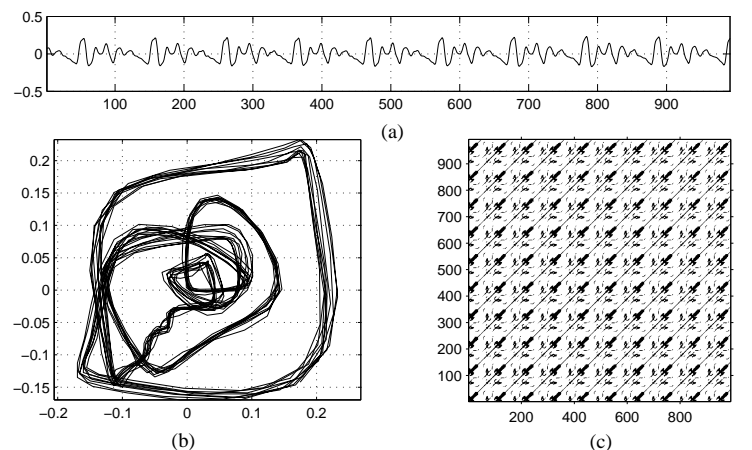

Figura 3: Sinal de voz saudável (a) Série temporal, (b) Retrato de fase e (c) Gráfico de recorrência; $T=1.000, m=3, \tau=4$ e $\varepsilon=0,1$ (norma euclidiana).
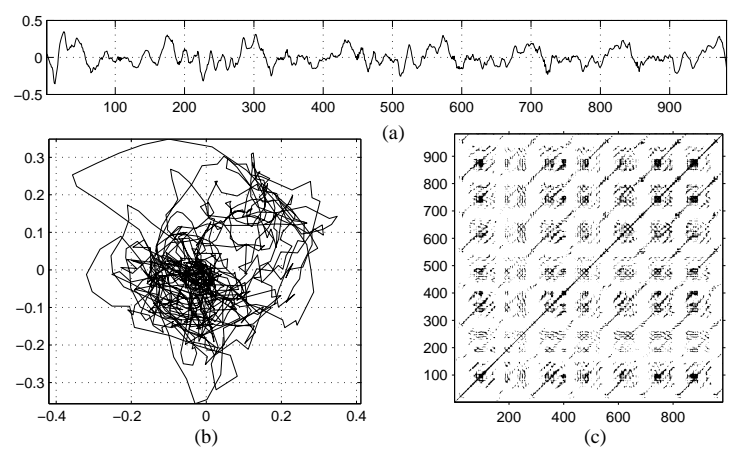

Figura 4: sinal de voz patológica (a) Série temporal, (b) Retrato de fase e (c) Gráfico de recorrência; $T=1.000, m=3, \tau=9$ e $\varepsilon=0,1$ (norma euclidiana).

dos e tornando-a menos subjetiva, Zbilut e Webber (Webber-Jr. and Zbilut, 1994) desenvolveram as primeiras medidas de quantificação de recorrência. As medidas inicialmente propostas estão relacionadas à densidade dos pontos de recorrência e às estruturas diagonais presentes nos gráficos de recorrência. Posteriormente, Marwan (Marwan, 2003) propôs outras medidas de recorrência, desta vez baseadas nas estruturas verticais (ou horizontais).

Algumas das medidas propostas por Zbilut, Webber e Marwan foram avaliadas em um trabalho anterior (de A Costa et al., 2012). Neste trabalho são analisadas as medidas de quantificação de recorrência denominadas de Transitividade, Comprimento médio das linhas diagonais, Tempo de recorrência de tipo 1 e Tempo de recorrência de tipo 2.

A medida Transitividade $(T R A N S)$ é baseada na teoria das redes recorrentes complexas (Marwan et al., 2009), e é uma espécie de taxa de recorrência local, local clustering, sendo

dada por:

$$
\operatorname{TRANS}=\frac{\sum_{i, j, k=1}^{N} \mathcal{R}_{i, j}^{m, \varepsilon} \mathcal{R}_{i, k}^{m, \varepsilon} \mathcal{R}_{k, i}^{m, \varepsilon}}{\sum_{i, j, k=1}^{N} \mathcal{R}_{i, j}^{m, \varepsilon} \mathcal{R}_{k, i}^{m, \varepsilon}}
$$

O Comprimento médio das linhas diagonais $\left(L_{m e d}\right)$ é uma medida do tempo médio em que dois segmentos de uma trajetória estão próximos um do outro, podendo ser interpretado como o tempo médio de predição. É calculado da seguinte maneira:

$$
L_{\text {med }}=\frac{\sum_{l=l_{\text {min }}}^{N} l \times P^{\varepsilon}(l)}{\sum_{l=l_{\text {min }}}^{N} P^{\varepsilon}(l)},
$$

em que $P^{\varepsilon}(l)$ representa a distribuição de frequência dos comprimentos $l$ das estruturas diagonais, e $l_{\text {min }}$ é o número mínimo de estruturas diagonais que se deseja contabilizar dentro do gráfico de recorrência.

O conjunto de todos os pontos recorrentes dentro de um determinado raio de vizinhança constitui os pontos de recorrência de tipo 1 . Os pontos de recorrência de tipo 2 são os primeiros pontos da trajetória do espaço de fases que caem nessa vizinhança. Dessa forma, é possível estimar dois tipos de tempo de recorrência, os quais estão relacionados a transições de energia na dinâmica do sistema (Gao and Cai, 2000).

O tempo de recorrência de tipo $1\left(T^{1}\right)$ indica a distância entre o ponto recorrente $\vec{\xi}_{j}$ e o ponto referência do raio de vizinhança, $\vec{\xi}_{i}$ (Marwan, 2003), e é dado por:

$$
T_{j}^{1}=\left|\left\{i, j: \vec{\xi}_{i}, \vec{\xi}_{j} \in \mathcal{R}_{i}\right\}\right|,
$$

em que $\mathcal{R}_{i}$ representa os pontos de recorrência na vizinhança de $\vec{\xi}_{i}$.

O tempo de recorrência de tipo $2\left(T^{2}\right)$ pode indicar com mais precisão do que $T^{1}$ transições de energia significantemente baixas (Gao et al., 2003). É definido como a distância entre o primeiro ponto recorrente da trajetória do espaço de fases, $\vec{\xi}_{j}$, e o ponto referência do raio de vizinhança, $\vec{\xi}_{i}$, e é dado por:

$$
T_{j}^{2}=\left|\left\{i, j: \vec{\xi}_{i}, \vec{\xi}_{j} \in \mathcal{R}_{i}, \vec{\xi}_{j-1} \notin \mathcal{R}_{i}\right\}\right|
$$

\subsection{Materiais Utilizados e Análise Realizada}

Neste trabalho são analisados 167 sinais de vozes, oriundos da Disordered Voice Database, Model 4337, desenvoldida pelo Kay Massachusetts Eye and Ear Infirmary (MEEI) Voice and Speech Lab (Elemetrics, 1994). Desse total, 53 sinais são de vozes saudáveis e 114 sinais são de vozes afetadas por patologias laríngeas (52 sinais de vozes afetadas por paralisia nas pregas vocais, 44 sinais de vozes afetadas por edema de Reinke, e 18 sinais 
de vozes afetadas por nódulos vocais). A taxa de amostragem de todos os sinais é de 25000 amos$\operatorname{tras} / \mathrm{s}$.

Para uma análise a curto intervalo de tempo, cada sinal é particionado em segmentos consecutivos de $32 \mathrm{~ms}$ (quadros de 800 amostras). De cada segmento são extraídos os parâmetros de imersão $(\tau$ e $m)$ e as medidas de quantificação de recorrência, utilizando a toolbox CRP (Cross Recurrence Plots) (Marwan and PIK, 2013). As medidas são extraídas de modo que a Taxa de Recorrência não ultrapasse $1 \%$. Dessa forma, para cada segmento é obtido um valor para o raio de vizinhança, $\varepsilon$, que mantenha esse patamar de Taxa de Recorrência.

As quatro caraterísticas de recorrência, TRANS, $L_{m e d}, T^{1}$ e $T^{2}$, são investigadas na discriminação entre os sinais de vozes saudáveis e os sinais de vozes patológicoa. O valores obtidos dessas medidas para cada sinal considerado são submetidos a uma análise estatística, com o teste de hipótese não paramétrico de Mann-Whitney para o caso de duas amostras independentes, considerando um nível de significância de 0,05, a fim de investigar o potencial discriminativo das medidas trabalhadas.

Para fins de classificação dos sinais como saudáveis ou patológicos, é empregada a Análise Discriminante Quadrática (QDA - Quadratic Discriminant Analysis), a qual é realizada com validação cruzada e 10 subconjuntos de forma estratificada.

\section{Resultados}

Na Tabela 1 estão apresentados os valores médios e os respectivos valores de desvio padrão para cada uma das medidas empregadas considerando os sinais saudáveis (SDL) e patológicos (PTL).

Tabela 1: Valor médio e o desvio-padrão das medidas de quantificação de recorrência utilizadas.

\begin{tabular}{|c|c|c|}
\hline Medidas & SDL & PTL \\
\hline TRANS & $0,61 \pm 0,02$ & $0,50 \pm 0,07$ \\
\hline$L_{\text {med }}$ & $10,39 \pm 2,72$ & $4,94 \pm 2,02$ \\
\hline$T^{1}$ & $94,64 \pm 7,66$ & $68,81 \pm 18,48$ \\
\hline$T^{2}$ & $146,73 \pm 22,21$ & $133,70 \pm 31,27$ \\
\hline
\end{tabular}

Após a aplicação do teste de hipótese não paramétrico de Mann-Whitney, os resultados revelaram que todas as quatro medidas avaliadas apresentam diferenças estatisticamente significativas $(\mathrm{P}<0,001)$ entre os dois grupos de sinais de voz considerados.

Na Tabela 2 são apresentados os valores médios e desvios padrão obtidos da análise de desempenho da classificação realizada de forma individual. As medidas de desempenho utilizadas foram a Taxa de Acurácia (Ac), Taxa de Falsos Positivos (FP) e Taxa de Falsos Negativos (FN).
Tabela 2: Valor médio e desvio padrão do desempenho da classificação realizada nas medidas de forma individual.

\begin{tabular}{|c|c|c|c|}
\hline Medidas & Ac (\%) & FP (\%) & FN (\%) \\
\hline TRANS & $76,95 \pm 1,61$ & $10,57 \pm 1,65$ & $28,86 \pm 2,05$ \\
\hline$L_{\text {med }}$ & $84,67 \pm 1,08$ & $25,41 \pm 2,22$ & $10,64 \pm 0,93$ \\
\hline$T^{\perp}$ & $77,74 \pm 1,61$ & $10,50 \pm 2,20$ & $27,72 \pm 2,66$ \\
\hline$T^{2}$ & $50,40 \pm 1,87$ & $22,58 \pm 3,50$ & $62,16 \pm 2,61$ \\
\hline
\end{tabular}

Após a verificação do potencial de cada uma das medidas na classificação entre os grupos de vozes saudáveis e patológicas, foram realizadas os testes com vetores formados a partir de todas as combinações possíveis das características avaliadas.

Na Tabela 3 são apresentados as melhores taxas obtidas para as medidas de desempenho na classificação realizada de forma combinada (COMB).

Tabela 3: Valor médio e desvio padrão dos melhores desempenho da classificação realizada nas medidas de forma combinada (COMB).

\begin{tabular}{|c|c|c|c|}
\hline COMB & Ac (\%) & FP (\%) & FN (\%) \\
\hline 2 a 2 & $88,50 \pm 1,67$ & $5,47 \pm 2,10$ & $14,30 \pm 2,37$ \\
3 a 3 & $89,44 \pm 1,02$ & $4,97 \pm 1,93$ & $13,16 \pm 1,33$ \\
Todas & $90,14 \pm 1,33$ & $6,48 \pm 2,14$ & $11,43 \pm 1,60$ \\
\hline
\end{tabular}

Na classificação usando vetores formados pela combinação de duas diferentes características, o melhor desempenho obtido foi com as medidas: Tempo de recorrência de tipo 1 e Transitividade. $\mathrm{Na}$ classificação com três características combinadas, o melhor desempenho foi obtido com as medidas: Tempo de recorrência de tipo 1, Tempo de recorrência de tipo 2 e Transitividade.

\section{Discussões}

As medidas de recorrência avaliadas confirmaram, no processo de classificação, os indícios apresentados no testes estatísticos realizados. Ou seja, a capacidade das medidas investigadas em discriminar os dois grupos de sinais de voz considerados (saudável e patológico). Entre as características consideradas de forma individual, a medida do Comprimento médio das linhas diagonais $\left(L_{m e d}\right)$ apresentou melhores taxas de classificação entre os dois grupos, em relação as demais. Como esperado, a utilização de vetores, formados por meio da combinação adequada de caraterísticas, oferece melhores taxas de desempenho quando comparadas aos obtidos com qualquer característica de forma individual. 


\section{Conclusão}

Os resultados do processo classificação apresentados sugerem que as medidas de recorrência analisadas podem ser utilizadas na construção de vetores característicos visando a discriminação entre sinais de vozes saudáveis e patológicas. Espera-se ainda que esses medidas possam oferecer taxas de desempenho ainda mais elevadas no caso de grupos de sinais patológicos mais específicos, uma vez que o grupo de sinais de vozes patológicas analisado é composto por três diferentes patologias, inclusive, de naturezas diferentes, ou seja nódulos e edema de origem orgânica e paralisia de origem neurológica. Além disso, entre os sinais analisados há diferentes graus de severidade da patologia, indivíduos de diversas faixas etárias e dos dois gêneros.

\section{Agradecimentos}

Os autores agradecem à CAPES pelo financiamento parcial da pesquisa, e à UFCG pela base de dados com os sinais de voz.

\section{Referências}

Behlau, M. (2001). A Voz do Especialista, Vol. 1, Revinter, Rio de Janeiro.

de A Costa, W., Assis, F. M., Neto, B. G. A., Costa, S. C. and Vieira, V. J. D. (2012). Pathological voice assessment by recurrence quantification analysis, Biosignals and $B i$ orobotics Conference (BRC), 2012 ISSNIP, IEEE, pp. 1-6.

de Oliveira Rosa, M. (2002). Laringe digital, PhD thesis.

Deller, J. R., Proakis, J. G. and Hansen, J. H. (2000). Discrete-time processing of speech signals, Ieee New York, NY, USA:

Eckmann, J. P., Kamphorst, S. O. and Ruelle, D. (1987). Recurrence plots of dynamical systems, Europhys. Letters 4(9): 973-977. DOI: $10.1209 / 0295-5075 / 4 / 9 / 004$

Elemetrics, K. (1994). Kay elemetrics corp. disordered voice database, Model 4337, 03 Ed.

Fant, G. (2004). Speech acoustics and phonetics: Selected writings, Vol. 24, Springer.

Fiedler-Ferrara, N. and do Prado, C. C. (1994). Caos: uma introdução, Edgar Blucher.

Fraser, A. M. and Swinney, H. L. (1986). Independent coordinates for strange attractors from mutual information, Physical review A 33(2): 1134. DOI: 10.1103/PhysRevA.33.1134
Gao, J. and Cai, H. (2000). On the structures and quantification of recurrence plots, Physics Letters A 270(1): 75-87. DOI: 10.1016/S0375-9601(00)00304-2

Gao, J., Cao, Y., Gu, L., Harris, J. and Principe, J. (2003). Detection of weak transitions in signal dynamics using recurrence time statistics, Physics Letters A 317(1): 64-72. DOI: 10.1016/j.physleta.2003.08.018

Hausdorff, F. (1919). Dimension und äussers mass, Mathematische Annalen 79: 157-179. DOI: 10.1007/BF01457179

Jiang, J., Zhang, Y. and McGilligan, C. (2006). Chaos in voice, from modeling to measurement, Journal of Voice 20 (1): 2-17. DOI: 10.1016/j.jvoice.2005.01.001

Kennel, M. B., Brown, R. and Abarbanel, H. D. I. (1992). Determining embedding dimension for phase-space reconstruction using a geometrical construction, Phy 45 (6): 34033411.

Kumar, A. and Mullick, S. K. (1996). Nonlinear dynamical analysis of speech, Journal Acous-tic Society of America 100(1): 615629. DOI: $10.1121 / 1.415886$

Marwan, N.(2003). Encounters With Neighbours - Current Developments of Concepts Based on Recurrence Plots and Their Applications, $\mathrm{PhD}$ thesis, University of Potsdam.

Marwan, N., Donges, J. F., Zou, Y., Donner, R. V. and Kurths, J. (2009). Complex network approach for recurrence analysis of time series, Physics Letters A 373(46): 4246-4254. DOI: 10.1016/j.physleta.2009.09.042

Marwan, N. and Kurths, J. (2004). Line structure in recurrence plots, Physics Letters $A$ 336: 349-357. DOI: 10.1016/j.physleta.2004.12.056

Marwan, N. and PIK, P. I. f. C. I. R. (2013). Cross recurrence plot toolbox for matlab, version 5.17 (r28.16). Disponível em http://tocsy.pikpotsdam.de/CRPtoolbox/index.html.

Acesso em fevereiro de 2013.

Savi, M. A. (2006). Dinâmica não-linear e Caos, E-papers.

Stemple, J. C., Glaze, L. E. and Gerdeman, B. K. (2000). Clinical voice pathology: Theory and management, Cengage Learning.

Takens, F. (1981). Detecting strange attractors in turbulence dynamical systems and turbulence, in D. Rand and L.-S. Young (eds), Lecture Notes in Mathematics, Berlin: Springer, pp. pp 366-381.

Webber-Jr., C. L. and Zbilut, J. P. (1994). Dynamical assessment of physiological systems and states using recurrence plot strategies, $J$. Appl. Physiol. 76: 965-973. 